\title{
Pandora: Single Unit Fully Coherent S and X Band Software Defined Radar
}

\author{
Filippo Scotti, Francesco Laghezza, Antonella Bogoni \\ *National Laboratory of Photonic Networks, CNIT \\ Via Moruzzi, 1, 56124 Pisa, ITALY \\ email: filippo.scotti@cnit.it
}

\begin{abstract}
A photonic-based coherent dual band radar system demonstrator, tested in an operative aerial scenario, is presented. Photonic technologies have been used for simultaneous generation and detection of radar signals in the $S$-and $X$-band. The sharing of a single transceiver for both the frequency bands allows for a perfect coherence among the generated waveforms.
\end{abstract}

\section{Introduction}

Since the second world war up to the present days a huge number of complex scenarios has been, and are, under observation with a flurry of radar systems, with different functionalities and objectives. Furthermore the possibility to include more than one functionality in a single radar unit allows to reach a better cognition of the same scene by properly using the software defined approach [1][2]. All the functionalities, as for example detection, tracking, imaging and so on, can benefit this concept thus enhancing the whole system performance, with also a strong costs reduction. Often, this approach requires the ability of the system to dynamically change the waveform parameters, to change the employed polarization, to perform frequency hopping in the same channel or among different bands and much more.

For instance, long range target detection and tracking are two key functionalities that typically require a proper frequency band selection. S-band radars are widely adopted for early warning applications, since the signals show a strong immunity against weather clutter, while radars operating in the X-band are often exploited to generate narrower beams for target tracking. Therefore, a multi-band radar can carry out the functions of different radars, reducing the costs and enhancing the performance. In fact, compared to the use of distinct conventional radar systems, coherent multi-band radars allow to enhance target classification and detection utilizing improved classification algorithms [1][2], and exploiting multispectral imaging of complex targets [3]. However the implementation of fully digital radar transceivers operating in different frequency bands is nowadays limited to carrier frequencies up to few $\mathrm{GHz}$ due to the bandwidth limitations of fast digital synthesizers (DDSs) and analog-to-digital converters (ADCs) [4].

In the last decades, researchers in the field of microwave photonics have been suggesting the possibility of exploiting the huge time-bandwidth product and flexibility of photonic technologies for the realization of wideband $\mathrm{RF}$ devices from few $\mathrm{MHz}$ up to several tens of $\mathrm{GHz}[5][6][7][8]$. To this purpose, the authors have been focusing in the introduction of photonics in both the generation [9] and the detection [10] of microwave signals, for wireless communications [11] as well as for radar systems [12]. The first photonics-based coherent radar system, realized under the ERC-funded project PHODIR, also proves the suitability of the photonic approach in the implementation and testing of operative radar systems [13]. The concept of a multi-band photonic transceiver has also been investigated by the authors [14]. In this paper the experimental validation of a photonic dual band coherent radar system, developed within the Pandora project, is presented. The demonstrator works simultaneously, coherently, and independently in the S- and X-band with a completely software defined 
approach. The basic design principles of the system architecture are presented in section II while targets detection results are detailed in section III.

\section{Principle of operation and system description}

The scheme of the developed architecture is sketched in Fig.1-left. The key element of the dual-band radar is a mode locked laser (MLL), which generates an extremely precise optical pulse train with a repetition rate $\mathrm{F}_{\mathrm{MLL}}=400 \mathrm{MHz}$. The laser optical spectrum is composed by a series of modes, phase locked to each other, spaced by $\mathrm{F}_{\mathrm{MLL}}$, as shown in Fig.1-A.

At the transmitter side, in order to generate two fully coherent modulated RF signals, a single DDS generates the combination of two waveforms at different intermediate frequencies $\mathrm{IF}_{1}=75 \mathrm{MHz}$ and $\mathrm{IF}_{2}=125 \mathrm{MHz}$, the spectrum of which is depicted in Fig. 1-B. The laser pulses are amplitude modulated by the IF waveforms via a Mach-Zehnder modulator (MZM). Due to the amplitude modulation, the intermediate-frequency signals are transferred as lower- and upper-sidebands around each mode of the MLL (Fig.1-C). When the optical signal thus modulated is detected with a photodiode (PD), all the spectral components are heterodyned together. This way, a replica of the signals from the DDS is obtained at every $\mathrm{kF}_{\mathrm{MLL}} \pm \mathrm{IF}_{\mathrm{n}}$, with $\mathrm{k}$ positive integer (Fig.1-D), up to the bandwidth of the photodiode (in this case, 12GHz). The output of the PD is so splitted onto two paths, where two electrical band-pass filters (BPFs) can extract the replicas of the signals upconverted to the desired carrier frequencies (CFs), as shown in Fig. 1-E. In details, the waveform generated at $\mathrm{IF}_{1}$ is upconverted to $\mathrm{CF}_{1}=9925 \mathrm{MHz}$, i.e. using the lower sideband of the $25^{\text {th }}$ harmonic, and the signal at $\mathrm{IF}_{2}$ is moved to $\mathrm{CF}_{2}=2925 \mathrm{MHz}$, i.e. the upper sideband of the $6^{\text {th }}$ laser harmonic. Once the two upconverted signals are isolated from the other harmonics, they are boosted up to a peak power of $20 \mathrm{~W}$ to be transmitted by two parabolic antennas with $30 \mathrm{dBi}$ gain and a field of view of about $10^{\circ}$.

The same antennas collect the echo signals which, opportunely filtered and amplified, feed the photonic receiver. Here they are coupled together and used to modulate the optical signal from the MLL by means of another MZM. This performs the optical sampling of the RF signals directly at carrier frequency (Fig.1-F), taking advantage of the extremely low time jitter of the MLL [10]. Finally, a 400Msample/s 12-bit ADC digitizes the photodetected signal. Since the transmitted waveforms have been generated together bellow the Nyquist frequency $\mathrm{F}_{\mathrm{MLI}} / 2$, the undersampling of the echo at $400 \mathrm{Msample} / \mathrm{s}$ doesn't produce overlapping of the different frequency bands, even if the whole signal span over $10 \mathrm{GHz}$.
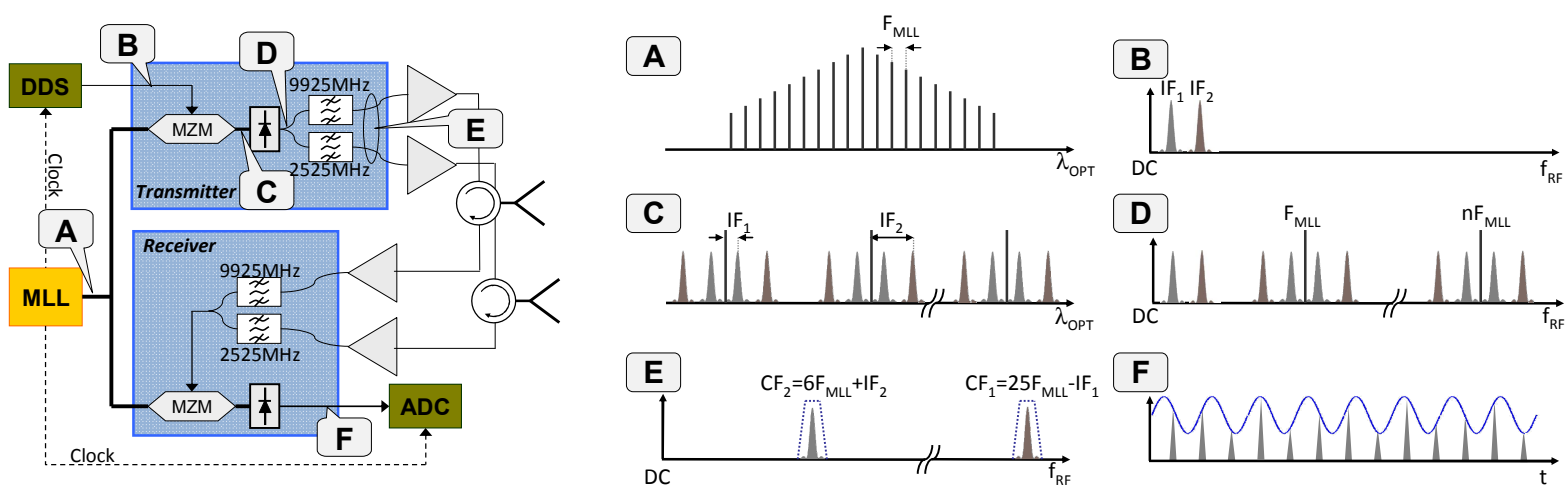

Figure 1. Left - Scheme of the dual-band radar system; Right - A) optical spectrum of the MLL, B) electrical spectrum of the modulating signal, C) optical spectrum after the MZM, D) electrical spectrum at the output of the photodiode, E) electrical spectrum after the filters, F) time domain representation of the echo signal sampled by the MLL. 


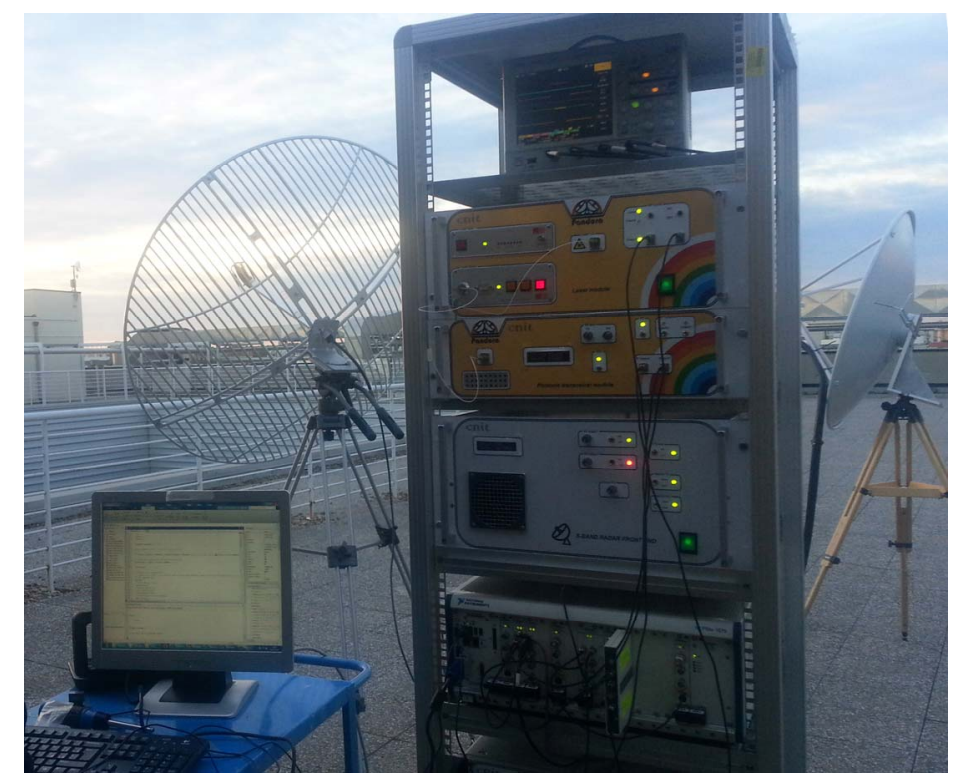

Figure 2. Picture of the PANDORA radar system during the preliminary field trial.

\section{Experimental results}

The photonic dual band coherent radar demonstrator has been tested targeting the non cooperative aerial traffic from the Pisa airport, nearby the laboratory (Fig.2). In order to prove the flexibility of the system, different waveforms have been employed. The results of two different radar acquisitions are depicted in Fig.3.

In the first case for the S-band channel the employed waveform was a standard pulse train with $3 \mu \mathrm{s}$ of pulse width (PW) and $10 \mathrm{kHz}$ of pulse repetition frequency (PRF). For the Xchannel a similar pulse train has been used with $3 \mu \mathrm{s}$ of PW and $8 \mathrm{kHz}$ of PRF. The range resolution for both the channels is $450 \mathrm{~m}$, while the Doppler frequency resolution was set to $100 \mathrm{~Hz}$, since the coherent integration time (CIT) has been set to $10 \mathrm{~ms}$. Under this conditions the coherent integrated pulses were about 100 and 80 for the S- and X- band respectively. In both the range-velocity maps shown in Fig.3-A a target about $2.6 \mathrm{~km}$ distant is clearly visible. The same distance is observed since the baseline of the two antenna systems is much less than the radar-to-target distance. The range profile of the S- and X-channel are depicted in Fig.3$\mathrm{B}$, and show a perfect superposition due to the same waveforms employed for both the cannels. The velocity profile is shown in Fig.3-C, and the better resolution $(1.5 \mathrm{~m} / \mathrm{sec})$ of the $\mathrm{X}$-band signal with respect to the S- channel $(6 \mathrm{~m} / \mathrm{sec})$ is clearly visible.

In the second case linearly chirped pulses were employed in both the channels. For the S-band the chirp frequency deviation was $4 \mathrm{MHz}$ over a PW of $3 \mu \mathrm{s}$ and $10 \mathrm{kHz}$ of PRF. For the Xchannel the employed chirp had a frequency deviation of $5 \mathrm{MHz}$ over a $\mathrm{PW}$ of $4 \mu \mathrm{s}$ and $10 \mathrm{kHz}$ of PRF. In this case the range resolutions were about $37.5 \mathrm{~m}$ and $30 \mathrm{~m}$ for the S- and Xchannel, respectively. The CIT was set to $50 \mathrm{~ms}$, thus reaching a Doppler frequency resolution of about $20 \mathrm{~Hz}$, integrating about 500 pulses. The range-velocity maps are shown in Fig.3-D where a strong echo peak is visible at a distance of about $3.6 \mathrm{~km}$ in both the maps. The superposition of the S- and X- channel range profiles is depicted in Fig.3-E, where the better range resolution of the $\mathrm{X}$ band waveform can be easily noted with respect to the $\mathrm{S}$ band. The velocity profiles, represented in Fig.3-F, clearly show that the increased CIT allow better velocity resolution in both the channels, even maintaining the better precision in the X-band. 
A
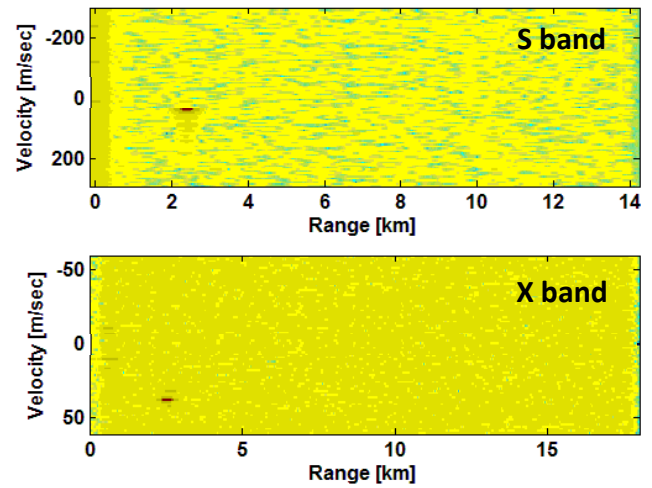

B

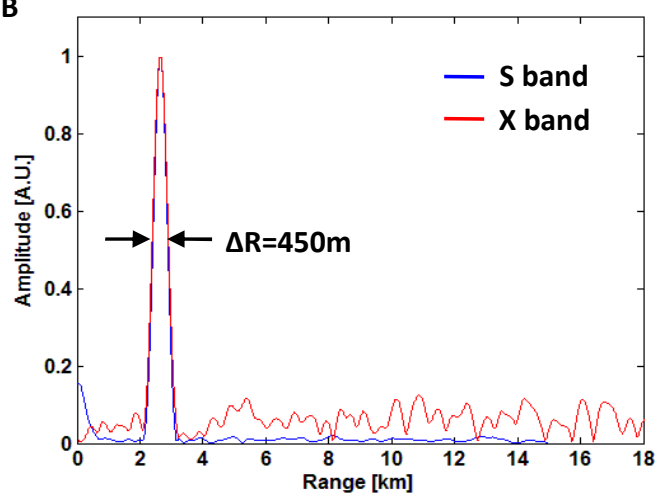

C

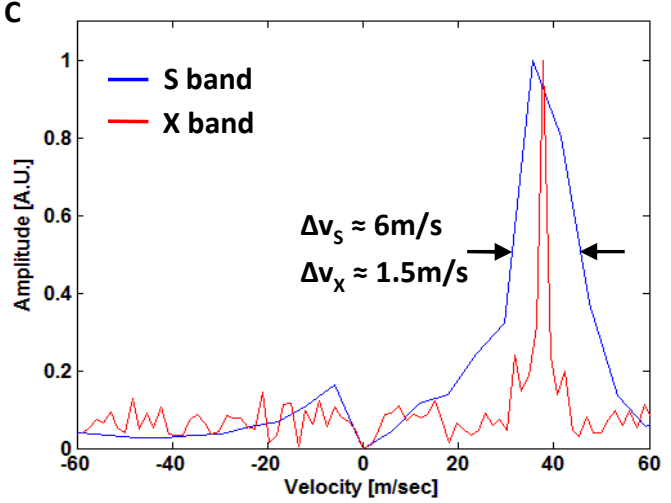

D
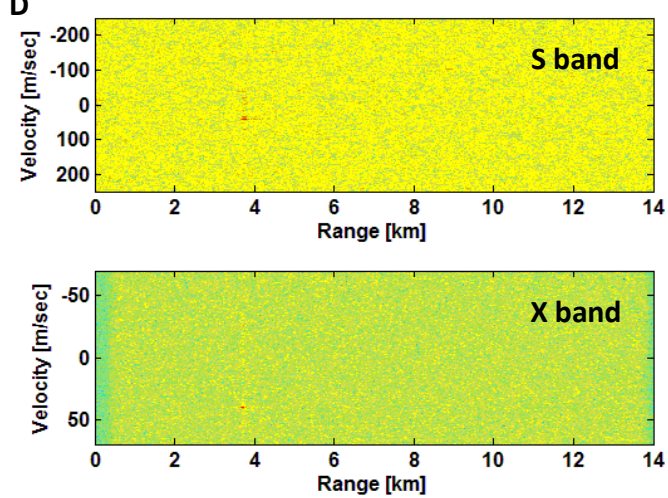

E

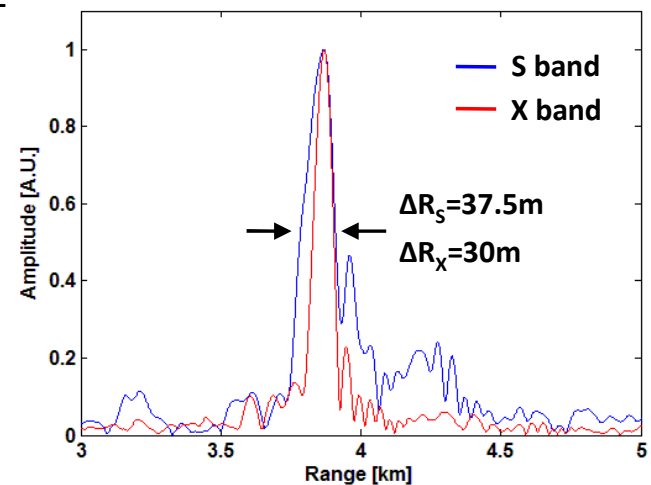

$\mathbf{F}$

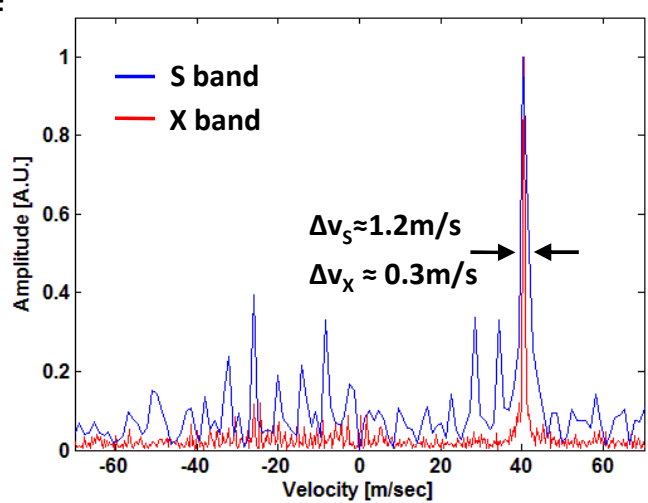

Figure 3. Range-velocity maps A), range profile B) and velocity profile C) of the first radar acquisition of non cooperative aerial target. Range-velocity maps D), range profile E) and velocity profile F) of the second radar acquisition of non cooperative aerial target.

\section{Conclusions}

In this paper, we have demonstrated the first photonics-aided dual-band radar demonstrator, capable of independently manage two different radar waveforms in two different frequency bands ( $\mathrm{S}$ and $\mathrm{X}$ ). At the transmitter side, multiple radar signals can be simultaneously generated by up-converting the waveforms generated at low IFs via a DDS. By using a single laser source, as reference multitone oscillator, the coherence among the generated waveforms has also been preserved opening new perspectives for advanced digital signal processing techniques.

Besides the received echo signals, in the two different bands, can be simultaneously sampled by a similar architecture and further digitized with a single ADC thus reducing the system complexity and cost. Experimental results, obtained during a preliminary field trial campaign, have been presented and discussed. 
This work has been carried on within EU projects RAPIDO, ROAM, PETRA, FiWin5G and national projects COMBINE (with the contribution of Ministry of Foreign Affairs, Directorate General for the Country Promotion) and MINOS (RBFR12N2T9).

\section{References:}

[1] M. Vespe, C. J. Baker, and H. D. Griffiths, "Automatic target recognition using multidiversity radar," IET Radar \& Sonar Navigation, vol.1, no.6, pp. 470-478, 2007.

[2] P. Van Dorp, R. Ebeling, and A. G. Huizing, "High resolution radar imaging using coherent multiband processing techniques," in IEEE Radar Conference, Washington DC, 2010, pp. 981-986.

[3] Xiao-lei Wei; Yong-an Zheng; Zhan-zhong Cui; Quan-li Wang, "Multi-band SAR Images Fusion Using the EM Algorithm in Contourlet Domain," Fuzzy Systems and Knowledge Discovery, 2007. FSKD 2007.

[4] A. Kaul, "Software defined radio: The transition from defense to commercial markets," in 2007 Software Defined Radio Technical Conf. Product Exposition, Nov. 2007.

[5] A. J. Seeds, C. H. Lee, E. E. Funk, and M. Naganuma, "Guest editorial: Microwave photonics," J. Lightw. Technol., vol. 21, no 12, pp. 2959-2960, Dec. 2003.

[6] C.H. Cox and E. I. Ackerman, "Microwave Photonics: past, present and future," in International Topical meeting on microwave photonics, 2008, pp. 9-11.

[7] J. Capmany and D. Novak, "Microwave Photonics combines two worlds," Nature Photonics, vol. 1, pp. 319-330, June 2007.

[8] J. Yao, "Microwave photonics," J. Lightw. Technol., vol. 27, no. 3, pp. 314-335, 2009.

[9] P. Ghelfi, F. Scotti, F. Laghezza, and A. Bogoni, "Phase Coding of RF Pulses in PhotonicsAided Frequency-Agile Coherent Radar Systems", IEEE J. Quantum Electron., vol.48, no.9, pp. 1151-1157, 2012.

[10] F. Laghezza, F. Scotti, P. Ghelfi, S. Pinna, and A. Bogoni; "Jitter-limited photonic analog-todigital converter with 7 effective bits for wideband radar applications," IEEE Radar Conference, Ottawa, 2013, pp.1-5.

[11] F. Laghezza, F. Scotti, P. Ghelfi, A. Bogoni, "Photonics-Assisted Multiband RF Transceiver for Wireless Communications," Lightwave Technology, Journal of, vol.32, no.16, pp.2896,2904, Aug.15, 152014.

[12] F. Scotti, et al., "In-field experiments of the first photonics-based software-defined coherent radar," J. Lightw. Technol., vol.32, no.20, pp. 3365-3372, Oct. 2014.

[13] P. Ghelfi, et al., "A fully photonics-based coherent radar system," Nature, vol.507, pp. 341345, Mar. 2014.

[14] F. Scotti, F. Laghezza, P. Ghelfi, S. Pinna, V. Vercesi, A. Bogoni, "Photonics-assisted dual band coherent radar system," EuRAD09-01, EURAD, Rome, Italy, Oct. 2014. 